\title{
Complex modes of vibration due to small-scale damping in a guitar top- plate
}

\author{
J. A. Torres, P. L. Rendón, R. R. Boullosa \\ Centro de Ciencias Aplicadas y Desarrollo Tecnológico, \\ Universidad Nacional Autónoma de México, México D.F. 04510 \\ jesusalejandrott@yahoo.com.mx, \\ pablo.rendon@ccadet.unam.mx \\ ricardo.ruiz@ccadet.unam.mx
}

\begin{abstract}
Modal analysis is one of the preeminent methods used by scientists and engineers to study vibrating structures. The frequency response functions obtained through this method, are, in general, complex-valued. There is, however, no agreed-upon interpretation given to the real and imaginary parts of these functions, even though it is acknowledged that their relative magnitude for different frequencies is related to the behaviour of the corresponding modes. A simple model is deduced to describe the shape of the spectrum associated with a finite-length time-signal. There is very good agreement between results obtained using this model and numerical results obtained for, in this case, the vibration of a guitar top-plate using finite element methods. One interpretation of the relative magnitudes of the real and imaginary parts of the frequency response functions is advanced. It is found that stationary-wave behaviour is associated with the dominance of the real or imaginary part; traveling-wave behaviour, on the other hand, occurs when the real and imaginary parts are of the same order of magnitude, as long as the scale of damping is large enough and resonance peaks in the spectrum are close enough.
\end{abstract}

Keywords: Complex modes, mode shapes, damped plates, guitar.

\section{RESUMEN}

El análisis modal es uno de los métodos utilizados con mayor frecuencia por científicos e ingenieros para estudiar estructuras vibrantes. Las funciones de respuesta de frecuencia obtenidas mediante este método tienen, en general, valores complejos. No existe, sin embargo, una interpretación universalmente aceptada asociada a las partes real e imaginaria de estas funciones, aún cuando se sabe que la magnitud relativa de estas cantidades para diferentes frecuencias está relacionada con el comportamiento de los distintos modos. Se obtiene un modelo sencillo que describe la forma del espectro asociado a una señal temporal de duración finita. Hay una muy buena concordancia enre los resultados obtenidos utilizando este modelo y aquellos obtenidos para, en este caso, la vibración de la tapa de una guitarra simulada a través de elementos finitos. Se propone una interpretación de las magnitudes relativas de las partes real e imaginaria de la función de respuesta de frecuencia. El comportamiento de onda estacionaria se asocia a la dominancia de la parte real o imaginaria; por otra parte, el comportamiento de onda viajera ocurre cuando las partes real e imaginaria son del mismo orden de magnitud, y siempre y cuando la escala del amortiguamiento sea lo suficientemente grande y los picos de resonancia en el espectro sean suficientemente cercanos.

\section{Introduction}

The vibrational behaviour of the different components of stringed instruments has been the subject of quite a few studies in the past decades, especially where low frequencies are concerned. This is due partly to the observation that in the low-frequency régime the resonance frequencies are almost completely determined by single modes of vibration, and it is then easier to study them in relative isolation, but it is also due to the fact that for low frequencies the equation of motion for a vibrating plate may be approximated by a wave equation incorporating damping, in effect simulating a damped membrane. These results are discussed in detail by Fletcher and Rossing (1), and by Ahluwalia et al (2). Most of the research on vibrating plates and the radiation produced by the plates in the context of stringed instruments has focused on resonance frequencies. Authors such as Schelleng (3) have remarked, however, that damping introduces both blunting at resonance frequencies and small changes of phase between adjacent frequencies in the region between resonance peaks that do have an effect on radiation patterns. Studies realised on the violin by Bissinger (4) confirm the appearance of modes not determined solely by the resonance frequencies, and, furthermore, concluded that the presence of these modes altered the perception of sound radiated from the instrument components under study. These modes have come to be known as complex modes, and are still considered difficult to identify. Bissinger (4) goes as far as to suggest that these modes can only be properly distinguished from normal modes through observation of an animation of the vi- 
bration of the structure. In more general terms, Spiekermann and Radcliffe $(5 ; 6)$ identify absorptive boundary conditions with a traveling-wave response and reflective boundary conditions with a standing-wave response, while noting that real conditions are necessarily associated to a mixed response. They then proceed to decompose both analytically (5) and experimentally (6) the total acoustic response associated with mixed boundary conditions into propagating and standing-wave components. A quantitative measure of the relationship between the two distinct ideal responses is given by scaling factors and phase angles obtained from the total mixed response. One of the main objectives of this paper is to show the manner in which even a harmonic signal acquires a complex mode structure after Fourier transformation, and how damping plays an essential part in determining to what extent these complex modes resemble either standing waves or traveling waves.

\subsection{The motion of vibrating plates: modal analysis and Finite-Element Methods}

Since the equations describing the motion of a vibrating plate are difficult to solve analytically for an object with a complex geometry, such as a guitar plate, the dynamic properties of these structures are often described in terms of what are called modal parameters: natural frequency, damping factor, modal mass, and mode shape. This technique is called modal analysis, and can be implemented mathematically by attempting to uncouple the structural equations of motion so that the resulting equations can then be solved individually. If these equations cannot be solved exactly, then numerical approximations are generally used. One such method of approximation is the Finite Elements Method (FEM), which consists of breaking down the object into a large number of smaller, more manageable (finite) elements. Then the complex equations that describe the behaviour of a structure can often be reduced to a set of linear matrix equations which can be solved using standard matrix algebra techniques. The manner in which we implemented the FEM scheme for the present work is described in detail by Inman (7) and Hatch (8).

The FEM representation of a continuous structure leads to the matrix system

$$
\mathbf{M} \ddot{\mathbf{x}}+\mathbf{C} \dot{\mathbf{x}}+\mathbf{K x}=\mathbf{f}(t),
$$

where $\mathbf{M}, \mathbf{C}$ and $\mathbf{K}$ are the $n \times n$ matrices containing mass, damping and stiffness terms respectively, and which are constructed from individual element matrices. The forcing system is an $n \times 1$ vector $f(t)$ and the displacement responses are denoted by the $n \times 1$ vector $\mathrm{x}$. The first step in modal analysis is carried out when we make the right-hand side of equation (1) equal to zero, in order to obtain the natural frequencies and the undamped mode shapes of the structure. Modes corresponding to these natural frequencies are called normal modes, the shapes of which are, as expected, orthogonal to all other mode shapes of vibration for the system. Thus, all points in the structure reach maximum amplitude of vibration, in either direction, at the same time. From a mathematical standpoint, normal modes represent the solution of an undamped system, and the normal-mode vectors contain a real number for each motional degree of freedom (DOF) studied. However, most realistic descriptions of vibrating structures do contemplate some form of damping, and in general this leads to the appearance of complex modes, where phase angles need not necessarily be either $0^{\circ}$ or $180^{\circ}(9)$. The maximum amplitude of vibration may then occur at different times for different points on the structure, in the same manner as is observed, for example, in a travelingwave pattern.

The frequency-response function (FRF) describes the structural response to an applied force as a function of frequency. In this paper, the response is given in terms of the velocity of a point on the structure, so that the measured quantity would be the mobility (velocity/force). For normal modes, the imaginary part of the FRF is zero at resonances, and the real part attains a maximum value; for complex modes, however, this is no longer valid and the imaginary part of the FRF is no longer zero. A classical treatment of the interpretation of the real and imaginary parts of the velocity response in a mode with complex eigenvalues is included in Newland (10). Some authors, such as Marshall (11), give the FRF in terms of inertance (acceleration/force), and in that case the real part of the FRF would be zero at resonances, and not the imaginary part. Marshall provides a very complete description of the technique of modal analysis in the context of studying the vibrational behaviour of a violin, and also remarks that it is only possible to excite a single resonance frequency if this frequency is sufficiently separated from other resonance frequencies and if damping is low enough to prevent coupling from occurring. It has become standard practice to accept 
the above-mentioned assumptions hold in most situations, and this has led most authors to plot the imaginary parts of FRFs (when given in terms of inertance) to illustrate vibrational behaviour - this, of course, is due to the dominance of the imaginary part of the FRF at resonance peaks. A more comprehensive portrait of vibration would very likely be achieved using complex modes. As the very definition of complex modes seems to be subject to different interpretations, we include in the following section a brief discussion of what are referred to as complex modes in this paper.

\subsection{Complex modes}

Wave propagation can be described in terms of complex solutions to wave equations, as is well-known. Take the traveling-wave solution to the undamped linear one-dimensional wave equation given by $y(x, t)=$ $\sin k(x-c t)$, where $y$ is the perturbation amplitude, $x$ is displacement, $t$ is time, $k$ is the wave number, and $c$ is the local sound speed. This solution can then also be written as $y(x, t)=\operatorname{Re}[\exp (i \omega t) \exp (i k x)]$, where $c=\omega / k$. Feeny (12) takes the form of this solution as a starting point in order to define complex modes as $\mathbf{x}(\mathbf{t})=\mathbf{e}^{\alpha \mathbf{t}} \mathbf{a}$, where $\mathbf{x}$ is a position vector, the same as $\mathbf{a}=\mathbf{u}+\mathbf{i v}$, and $\alpha=\gamma+i \omega$, with $\gamma, \omega$, $\mathbf{u}$, and $\mathbf{v}$ real. The real part of this complex mode is then easily seen to be

$$
\operatorname{Re}[\mathbf{x}(\mathbf{t})]=\mathbf{e}^{\gamma \mathbf{t}}[\cos (\omega \mathbf{t}) \mathbf{u}-\sin (\omega \mathbf{t}) \mathbf{v}] .
$$

Thus, $\mathrm{x}$ will, in most cases, continually oscillate between the values of $\mathbf{u}$ and $\mathbf{v}$ due to the phase difference between the cosine and sine functions above. Feeny observes that the relative sizes and degree of independence of $\mathbf{u}$ and $\mathbf{v}$ will determine whether, from a qualitative standpoint, the motion appears to be of a traveling-wave type or of a standing-wave type. He goes on to define an index which characterises these transitions quantitatively. For our purposes, however, it will be sufficient to observe that both extremes are obtainable from the single form above. If, for example, we were to consider $\mathbf{u}=\mathbf{v}$ in equation (2), we would obtain

$$
\operatorname{Re}[\mathbf{x}(\mathbf{t})]=\sqrt{\mathbf{2}} \mathbf{e}^{\gamma \mathbf{t}} \cos (\omega \mathbf{t}-\pi / \mathbf{4}) \mathbf{u},
$$

which clearly represents a standing wave. Now if either $\mathbf{u}=\mathbf{0}$ or $\mathbf{v}=\mathbf{0}$ we would recover a travelingwave solution with the same form as that introduced at the beginning of this section. In this interpretation, all modes are complex, even those normally referred to as normal modes - these simply correspond to one of the two extremes described above. As we discussed in section 1.1, it is, however, common practice to identify modes in the spectral as well as in the temporal domain, in particular through the FRF. In this paper we will discuss the interpretation of the real and imaginary parts of the FRF, and observe that the two extreme types of complex modes discussed above may also be distinguished from examination of the spectrum. The standing-wave or traveling-wave character of a measured signal will not only depend on the shape of the signal, but also on the length of the measurement interval, and, critically, on the scale of damping, as will be shown in the next section.

\section{An analytical model of the Fourier transform of a truncated signal}

As we have discussed in the previous sections, modal analysis provides a powerful way to study the vibrational behaviour of a plate with complicated geometry. The canonical equations used for this technique essentially describe coupled damped oscillators, and since for low frequencies the guitar plate may be assumed to vibrate very much in the same manner as a damped membrane, we consider here the simplest equation to combine wave propagation with linear damping, corresponding to a string with fixed-ends where a small amount of energy is gradually dissipated as the string vibrates. If $y=y(x, t)$ represents the vertical displacement of the string relative to the $x$-axis, then the equation is written as follows:

$$
\frac{1}{c^{2}} \frac{\partial^{2} y}{\partial t^{2}}+\epsilon \frac{\partial y}{\partial t}-\frac{\partial^{2} y}{\partial x^{2}}=0,
$$

where $x$ is position, $t$ is time, $c$ is the local sound speed, and $\epsilon$ is a non-negative constant associated to the small dissipation $(\epsilon \ll 1)$. The boundary conditions can be written, without loss of generality, for a string of length $L$, as:

$$
y(x=0, t)=y(x=L, t)=0 .
$$

The general form of the bounded solution to this equation can be easily obtained through separation of variables, and since $\epsilon \ll 1$ here it is written as:

$$
y(x, t)=\sin (k x) e^{c \epsilon t / 2} e^{ \pm i \alpha t},
$$

where $k=n \pi / L$, with $n$ any integer, and $\alpha=$ $\sqrt{\omega_{0}^{2}-\epsilon^{2} / 4}$, with $\omega_{0}=k / c$. Notice that this solution is of the general form given by Feeny for a 
complex mode in equation (2). Observe also that if $\epsilon=0$ the solution to equation (3) with boundary conditions given by (4) should correspond simply to the solution of the undamped linear wave equation, $y(x, t)=\sin (k x) \exp \left( \pm i \omega_{0} t\right)$. It is well known that the Fourier transform with respect to time of such a solution is real, having no imaginary part. Having stated in the previous section our interest in doing so, we will now calculate the Fourier transform with respect to time of solution (5) in order to examine the explicit rôle of damping in contributing toward the imaginary part of the spectrum.

We consider here all of the previous solutions to be given in terms of generalised functions, and the calculations involving Fourier transformations are then handled in that context as well. This is necessary in order to guarantee the existence of the transforms of the functions involved. For clarity's sake, the definition used here of the Fourier transform of generalised function $f(t)$ is

$$
\tilde{f}(\omega)=\mathcal{F}[f(t)]=\int_{-\infty}^{\infty} f(t) e^{-i \omega t} d t,
$$

and then its inverse is given by

$$
f(t)=\mathcal{F}^{-1}[\tilde{f}(\omega)]=\frac{1}{2 \pi} \int_{-\infty}^{\infty} \tilde{f}(t) e^{i \omega t} d \omega .
$$

A detailed discussion of generalised functions and of their Fourier transform is given in Kammler (13), as are the transforms for the following generalised functions, where $h(t)$ denotes the Heaviside function:

$$
\begin{aligned}
\mathcal{F}\left[e^{i \alpha t}\right] & =2 \pi \delta(\omega-\alpha), \\
\mathcal{F}[h(t)] & =\pi\left[\delta(\omega)-\frac{i}{\pi \omega}\right] .
\end{aligned}
$$

Using the convolution theorem for Fourier transforms we may then calculate the Fourier transform of a semiinfinite harmonic time signal (generality is not lost by assuming the signal starts at time zero):

$$
\begin{aligned}
\mathcal{F}\left[h(t) e^{i \alpha t}\right] & =\pi \int_{-\infty}^{\infty} \delta\left(\omega^{\prime}-\alpha\right)\left[\delta\left(\omega-\omega^{\prime}\right)\right. \\
& \left.-\frac{i}{\pi\left(\omega-\omega^{\prime}\right)}\right] d \omega^{\prime} \\
& =\pi\left[\delta(\omega-\alpha)-\frac{i}{\pi(\omega-\alpha)}\right] .
\end{aligned}
$$

It is interesting to observe that the Fourier transform of the semi-infinite time-harmonic solution already has a non-zero imaginary part, and that this accounts in some sense for the fact that measured time-harmonic signals never precisely yield delta funtions in the spectrum, as should be expected if the signals were of infinite duration. We now define

$$
v(t)=h(t) e^{-b t} e^{i \alpha t},
$$

where $b=c \epsilon / 2$ is a positive constant; it is then straightforward to identify $v(t)$ with the temporal part of the solution of the damped wave equation (3) where in addition to the boundary conditions we require that the system remain static until a particular time when the signal starts (and again, without loss of generality we may choose this time to be zero). This condition effectively corresponds to a situation where a signal is measured beginning at a certain time. Taking into account that

$$
\mathcal{F}\left[h(t) e^{-b t}\right]=\pi \delta(\omega)+\frac{1}{b+i \omega},
$$

and again using the convolution theorem, we obtain

$$
\begin{aligned}
\mathcal{F}[v(t)] & =\int_{-\infty}^{\infty} \delta\left(\omega^{\prime}-\alpha\right)\left[\pi \delta\left(\omega-\omega^{\prime}\right)\right. \\
& \left.+\frac{1}{b+i\left(\omega-\omega^{\prime}\right)}\right] d \omega^{\prime} \\
& =\pi \delta(\omega-\alpha)+\frac{1}{\frac{c \epsilon}{2}+i(\omega-\alpha)} .
\end{aligned}
$$

Notice that this solution tends, as $\epsilon \rightarrow 0$, precisely to the solution given in equation (8), which is the expected result. As we are especially interested in the imaginary part of this generalised function, we write it in the following, standard, form:

$$
\begin{aligned}
\mathcal{F}[v(t)] & =\left[\pi \delta(\omega-\alpha)+\frac{c \epsilon / 2}{c^{2} \epsilon^{2} / 4+(\omega-\alpha)^{2}}\right] \\
& -i\left[\frac{\omega-\alpha}{c^{2} \epsilon^{2} / 4+(\omega-\alpha)^{2}}\right] .
\end{aligned}
$$

We now consider two main cases:

(i) When considering frequencies very near or at a resonance frequency, $O(\omega-\alpha)=0$, and in that case the delta function term on the right hand side of (12) clearly dominates the behaviour of the spectrum at that point. Notice that, for $\epsilon>0$, the other two terms do also contribute with inverse square decay (second 
real term) and inverse decay (imaginary term) away from the resonance frequency. This decay is clearly observed in the plots in the following section, where resonance frequencies do not appear as absolute discontinuities, but rather as relatively sharp peaks.

(ii) When frequencies are not in the immediate vicinity of a resonance frequency, say $O(\omega-\alpha) \geq 1$, the delta term becomes identically zero and makes no contribution to the spectrum. The contribution to the spectrum of the remaining two terms now also depends on the scale of the value of $\epsilon$. Firstly, as $\epsilon$ tends to zero, the second real term also tends to zero and only the imaginary term remains. However, for small but non-zero $\epsilon$ the relative magnitude of the remaining real term and the imaginary term is determined directly by $O(\omega-\alpha)$. If $O(\omega-\alpha)=1$, then the real term will be smaller than the imaginary term by an order of magnitude $\epsilon$, but if $O(\omega-\alpha) \geq 10$, then this same term will be smaller than the imaginary term by a larger order of magnitude, $\epsilon /(\omega-\alpha)$. Thus, as we consider frequencies further away from the resonance frequency, gradually the imaginary part of (12) comes to dominate almost completely the behaviour of the spectrum.

This analysis can be applied, in principle, to any vibrating system with small attenuation where the governing equation allows for separation of variables. Under these circumstances, we could, for example, substitute the second derivative with respect to position term in equation (3) with a more general Laplacian, with different boundary conditions, and the analysis of the temporal part of the solution that yields equation (12) would remain valid.

\section{Complex vibration modes of a guitar top-plate: analytical and simulated results}

Two distinct resonance frequencies of a system completely devoid of damping should, in theory, not interact. It is observed, though, that even in the presence of small-scale damping, these frequencies may interact depending on their proximity in the spectrum. Figure 1 below illustrates the shape of two resonance peaks calculated according to equation (12) separately; these frequencies correspond to the two lowest resonance peaks of the guitar top plate, which is the system we will refer to throughout this section. Notice that even though damping is non-zero for this simulation, the imaginary part of the FRF is zero for both resonance frequencies. Thus, damping seems, in this case, to only have the effect of reducing the maximum amplitude of the resonance peaks.

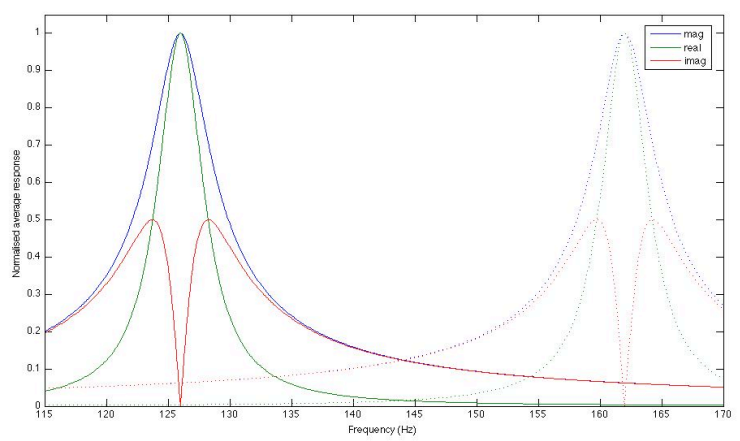

Figure 1: Plot of the real and imaginary parts of the normalised average response of a guitar top-plate calculated separately at the two lowest resonance peaks. Solid and dotted lines are used simply to distinguish between peaks.

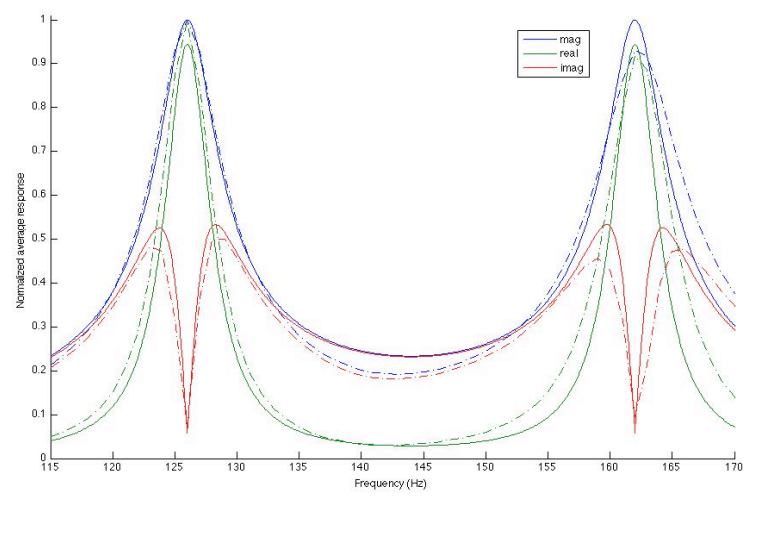

Figure 2: Plot of the real and imaginary parts of the normalised average response of a guitar top-plate calculated jointly at the two lowest resonance peaks. Solid lines denote the analytical result, and dotted lines the FEM simulation.

However, if we calculate the spectrum around these same resonance peaks, with the same damping coefficient as before, but considering the joint effect of both peaks, it becomes apparent that the imaginary part of the FRF is no longer zero at resonances, as can be seen in Figure 2. The real part still dominates at resonances, though, and the calculated FRF approximates very closely the normalised response 
calculated for the guitar top-plate using FEM with an equivalent damping factor. A Finite Element Method has been used to calculate the average of the absolute value of the velocity at all points on the mesh that covers the guitar top-plate, as a function of frequency; the scheme itself is described in detail by Torres and Boullosa (14). We observe then that not only does the introduction of damping have the effect of diminishing the amplitude of resonance peaks, but it also results, when taking into account interactions between the resonance peaks, in the spectrum consisting of complex values (meaning, in this context, not purely real or imaginary) even at resonances.

It is thus established that damping permits resonance peaks to interact if they are close enough together. In fact, as the scale of damping increases, the magnitude of the imaginary part of the response approaches the magnitude of the real part, as is shown in Figure 3. Indeed, the greater the damping the greater the scale of the interaction will be. When there is no damping, the imaginary part is identically zero, as predicted in section 2 .
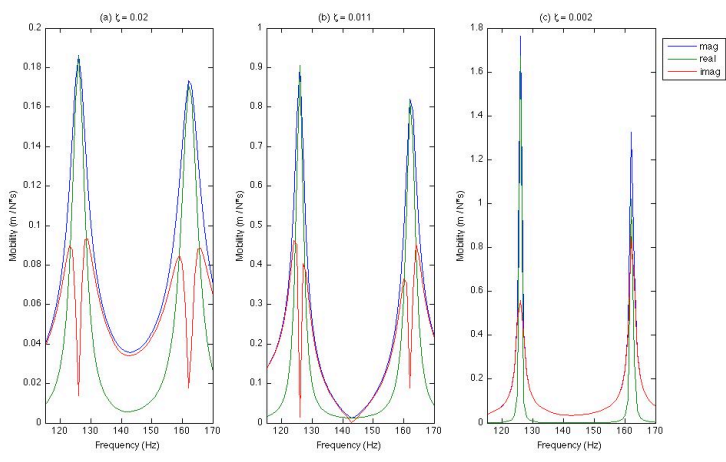

Figure 3: Plot of the simulated real and imaginary parts of the average response of a guitar top-plate at the two lowest resonance peaks for different values of the damping ratio $\zeta$.

In order to distinguish between traveling-wave and standing-wave behaviour in this example, we will introduce the notion of an Operational Deflection Shape (ODS), which is constructed from the collection of the FRFs corresponding to all points on the mesh which covers the guitar top-plate, and which gives the vibration pattern of the plate. Three ODSs are plotted in Figure 4, corresponding to $126 \mathrm{~Hz}, 140 \mathrm{~Hz}$ and 162 $\mathrm{Hz}$. The first and third frequencies are the resonance frequencies observed in Figure 2, with the second frequency falling between the resonance frequencies. We observe that for both resonance frequencies the principal contribution to the plate velocity is given by the real part of the ODS, as would be expected. Also, in both cases the imaginary part of the ODS is characterised by asymmetric dipoles, with opposite orientation due to a phase shift with respect to the axis of balance of the top-plate at around $126 \mathrm{~Hz}$. For these frequencies we essentially observe what is generally identified as normal-mode behaviour. For the intermediate frequency, however, we observe that the real part of the ODS exhibits a pole on the left side of the plate, while the imaginary part is dominated by a pole on the right side. Thus, due to the $90^{\circ}$ phase shift between real and imaginary parts, we conclude that a perturbation very much like a traveling-wave moves from left to right. This result is consistent with the fact that for this frequency, the real and imaginary parts of the FRF are of comparable order as seen, again, in Figure 2.
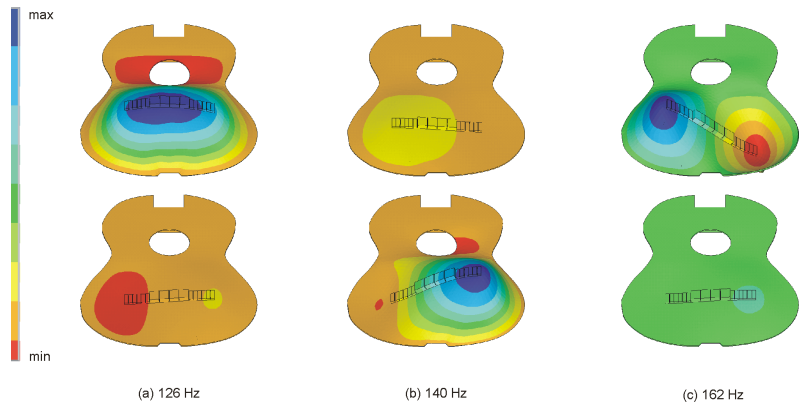

Figure 4: Normalised real and imaginary parts of the ODSs corresponding to (a) $126 \mathrm{~Hz}$, (b) $140 \mathrm{~Hz}$, (c) $162 \mathrm{~Hz}$.

\section{Conclusions}

In order to establish whether a particular mode behaves, in the traditional sense, more like a normal mode, or whether it is more closely associated to a traveling perturbation, Feeny, through a variation on complex orthogonal decomposition, proposes a quantitative measure of behaviour according to the timeevolution of this particular mode. However, a characterisation to be used in conjunction with modal analysis would preferably focus on the spectral, and not the 
temporal domain. A simple model is used to model the average spectrum of a vibrating structure, which will always be complex, even for harmonic signals, when the length of the signal is not infinite. Since modal shapes can be normalised, we are able to compare the low-frequency spectrum obtained using this model with that resulting from the FEM simulation of the average vibration of a whole guitar top-plate. The proximity of resonance peaks and the scale of damping are observed to determine the relationship between the real and imaginary parts of the FRFs, and then also establish whether the behaviour will tend to that of a stationary or a traveling wave. Thus, the modes commonly known as complex modes can, in general, be identified through study of the spectrum, and not only through the analysis of successive realisations of the ODSs for particular frequencies, as suggested by Bissinger (although they can also be pinpointed in this manner).

\section{Acknowledgements}

The authors would like to thank the Universidad Nacional Autónoma de México, which, through Coordinación de Estudios de Posgrado and project PAPIIT IN120008, has awarded financial support towards the production of this paper.

\section{References}

[1] Fletcher, N.H., and Rossing T.D., The Physics of Musical Instruments. Springer, New York, 1998.

[2] Ahluwalia, D.S., Kriegsmann, G.A., and Reiss, E.L., Scattering of low-frequency acoustic waves by baffled membranes and plates. J. Acoust. Soc. Am., 78(2):682-687, 1985.

[3] Schelleng, J.C., On the polarity of resonance. Catgut Acoust. Soc. Newsletter, (10):14-18, 1968.
[4] Bissinger, G., Some mechanical and acoustical consequences of the violin soundpost. J. Acoust. Soc. Am., 97(5):3154-3164, 1995.

[5] Spiekermann, C. E., and Radcliffe, C. J, Decomposing one-dimensional acoustic pressure response into propagating and standing waves. J. Acoust. Soc. Am., 84(4):1536-1541, 1988.

[6] Spiekermann, C. E., and Radcliffe, C. J, Stripping one-dimensional acoustic pressure response into propagating - and standing - wave components. J. Acoust. Soc. Am., 84(4):1542-1548, 1988.

[7] Inman, D. J., Engineering Vibration. Prentice Hall, London, 1994.

[8] Hatch, M. R., Vibration simulation using Matlab and Ansys. Chapman and Hall, London, 2001.

[9] Rossing, T. D., Modal analysis. In Thomas D. Rossing, editor, Springer Handbook of Acoustics, pages 1127-1138. Springer, New York, 2007.

[10] Newland, D.E., Mechanical vibration analysis and computation. Longman Scientific \& Technical, Harlow, England, 1989.

[11] Marshall, K.D., Modal analysis of a violin. J. Acoust. Soc. Am., 77(2):695-709, 1985.

[12] Feeny, B.F., A complex orthogonal decomposition for wave motion analysis. J. Sound Vibrat., 310:77-90, 2008.

[13] Kammler, D.W., A First Course in Fourier Analysis. Cambridge University Press, 2007.

[14] Torres, J.A., and Boullosa, R. R., Influence of the bridge on the vibrations of the top plate of a classical guitar. Applied Acoustics, 70(11-12):1371-1377, 2009. 


\section{Author biographies}

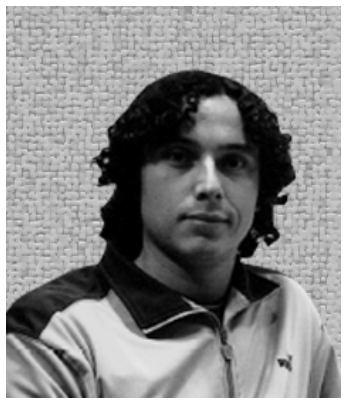

Jesús Alejandro TORRES-TORRES

Jesús Alejandro Torres Torres was born in 1981 in Mexico City. He graduated from the Instituto Tecnológico de Querétaro in Mechanical Engineering in 2003, beginning there his research of guitar physics. He received a Masters degree in 2006 from the School of Engineering at the Universidad Nacional Autónoma de México, where he is currently enrolled as a $\mathrm{PhD}$ student. His PhD dissertation, on the vibroacoustic behaviour of a guitar top plate, is to be defended in January 2010.

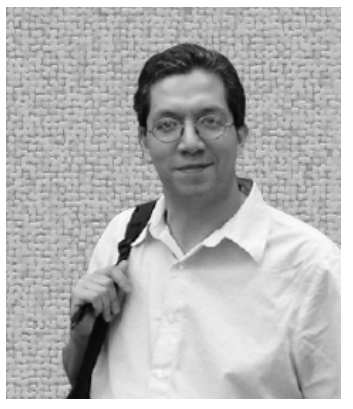

\section{Pablo Luis RENDÓN-GARRIDO}

Pablo Luis Rendón was born in Mexico City in 1971. He studied Physics at the School of Science at the Universidad Nacional Autónoma de México (UNAM). He went on to do Part III Mathematics at the Department of Applied Mathematics and Theoretical Physics at the University of Cambridge, where he also obtained his PhD in Applied Mathematics, working under the supervision of Prof. David G. Crighton, FRS. Afterwards, he participated in the Sonic Boom European Research (SOBER) programme, as a postdoctoral researcher based at the Université Pierre et Marie Curie (UPMC), Paris VI, working directly with the Research Director of the project, Dr. François Coulouvrat. Currently, he is a lecturer at the Centro de Ciencias Aplicadas y Desarrollo Tecnológico (CCADET), UNAM, where he conducts research and lectures both at undergraduate and graduate levels. Among his topics of interest are nonlinear acoustics, sonic booms, musical acoustics and water waves. 


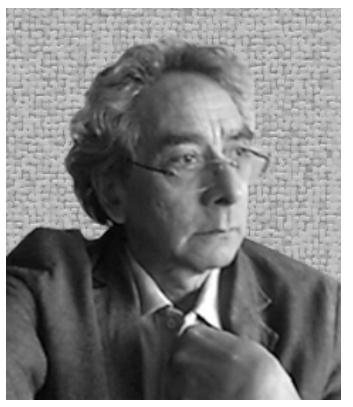

\section{Ricardo RUIZ-BOULLOSA}

$\mathrm{He}$ is a researcher since 1981 at the Acoustics and Vibration Group, CCADET-UNAM. Has a BSc in Physics (UNAM, 1972), an MSc degree in sound and vibration (University of Southampton, UK, 1981), and a PhD degree (CIICA-UAEM, Morelos, Mexico, 2006). Founder and chief executive of the Design Project of the CCADETs Acoustics Laboratory (1982-1988), head of the Acoustics Laboratory (1988-2000), and initiator of some of its research lines such as: acoustics of the guitar, urban noise, thermoacoustic sources, laser vibration measurements. Has authored and coauthored 35 papers in international journals (such as: Applied Acoustics, Acta Acustica, JASA, Applied Physics Letters) and 40 papers in national and international congresses, with around 75 citations to his work. He wrote the book "Laser: some demonstrations and simple experiments" (UNAM, 1979). He translated the book "Fundamentals of Acoustics," by Kinsler \& Fry, (John Wiley \& Sons, 1989). Has been thesis supervisor of $13 \mathrm{Bsc}, 4 \mathrm{MSc}$, and $1 \mathrm{PhD}$ thesis in Physics and Engineering. Reviewer in journals such as: Revista Mexicana de Física, Instrumentation \& Development, Acta Acustica united with Acustica. Member for several years of various internal academic committees in the CCADET. Member of the SNI (National Researcher's System - Level I, 1995-). Has drawing and painting as well as piano playing as spare-time activities. 